\title{
IRREDUCIBILITY CRITERIA FOR SUMS OF TWO RELATIVELY PRIME POLYNOMIALS
}

\author{
NICOLAE CIPRIAN BONCIOCAT, YANN BUGEAUD, MIHAI CIPU, AND MAURICE MIGNOTTE
}

\begin{abstract}
We provide irreducibility conditions for polynomials of the form $f(X)+p^{k} g(X)$, with $f$ and $g$ relatively prime polynomials with integer coefficients, $\operatorname{deg} f<\operatorname{deg} g, p$ a prime number and $k$ a positive integer. In particular, we prove that if $k$ is prime to $\operatorname{deg} g-\operatorname{deg} f$ and $p^{k}$ exceeds a certain bound depending on the coefficients of $f$ and $g$, then $f(X)+p^{k} g(X)$ is irreducible over $\mathbb{Q}$.
\end{abstract}

\section{INTRODUCTION}

The sum of two relatively prime polynomials is by no means necessarily irreducible, as one can easily check by considering relatively prime polynomials of small degree having coefficients in arbitrary unique factorization domains. However some linear combinations of two relatively prime polynomials, say $n_{1} f(X)+n_{2} g(X)$, prove to be irreducible, provided some conditions on the factorization of $n_{1}$ and $n_{2}$ are satisfied. For instance, there are several recent irreducibility criteria for polynomials of the form $f(X)+p g(X)$, where $f$ and $g$ are relatively prime polynomials with rational coefficients, and $p$ is a sufficiently large prime number. In [5] Cavachi proved that for any relatively prime polynomials $f(X), g(X) \in$ $\mathbb{Q}[X]$ with $\operatorname{deg} f<\operatorname{deg} g$, the polynomial $f(X)+p g(X)$ is irreducible over $\mathbb{Q}$ for all but finitely many prime numbers $p$. In [6] this result has been improved by providing an explicit lower bound $p_{0}$ depending on $f$ and $g$, such that for all primes $p>p_{0}$, the polynomial $f(X)+p g(X)$ is irreducible over $\mathbb{Q}$. The method in [6] was adapted in [2] in order to provide sharper bounds $p_{0}$ as well as explicit upper bounds for the total number of factors over $\mathbb{Q}$ of linear combinations of the form $n_{1} f(X)+n_{2} g(X)$, where $f$ and $g$ are relatively prime polynomials with $\operatorname{deg} f \leq \operatorname{deg} g$, and $n_{1}$ and $n_{2}$ are non-zero integers with absolute value of $n_{2} / n_{1}$ exceeding an explicit lower bound. Similar results have been also provided for compositions of polynomials with integer coefficient [4] and for multiplicative convolutions of polynomials with integer coefficients [3].

The aim of this paper is to complement the results in [2] and [6], by providing irreducibility conditions for some classes of polynomials of the form $f(X)+p^{k} g(X)$, where $f$ and $g$ are relatively prime polynomials with integer coefficients, $p$ is a prime number and $k$ a positive integer.

2000 Mathematics Subject Classification. Primary 11R09; Secondary 11C08.

Key words and phrases. irreducible polynomials, prime numbers, resultant. 
For any polynomial $f(X)=a_{0}+a_{1} X+\cdots+a_{n} X^{n} \in \mathbb{Z}[X]$ of degree $n$, we set

$$
H(f)=\max \left\{\left|a_{0}\right|,\left|a_{1}\right|, \ldots,\left|a_{n}\right|\right\}
$$

the usual height of $f$.

We will first prove the following effective result that provides explicit conditions on $k$ and explicit lower bounds for $p^{k}$ depending on $f$ and $g$, that ensure the irreducibility of the polynomial $f(X)+p^{k} g(X)$.

Theorem 1.1. Let $f, g \in \mathbb{Z}[X]$ be two relatively prime polynomials with $\operatorname{deg} g=n$ and $\operatorname{deg} f=n-d, d \geq 1$. Then for any prime number $p$ that divides none of the leading coefficients of $f$ and $g$, and any positive integer $k$ prime to $d$ such that

$$
p^{k} \geq\left(2+\frac{1}{2^{n+1-d} H(g)^{n+1}}\right)^{n+1-d} H(f) H(g)^{n}-\frac{H(f)}{H(g)},
$$

the polynomial $f(X)+p^{k} g(X)$ is irreducible over $\mathbb{Q}$.

In particular, we obtain the following two corollaries.

Corollary 1.2. Let $f, g \in \mathbb{Z}[X]$ be two relatively prime polynomials with $\operatorname{deg} g=n$ and $\operatorname{deg} f=n-d, d \geq 1$, and let $k$ be a positive integer prime to $d$. Then the polynomial $f(X)+p^{k} g(X)$ is irreducible over $\mathbb{Q}$ for all but finitely many prime numbers $p$.

Corollary 1.3. Let $f, g \in \mathbb{Z}[X]$ be two relatively prime polynomials with $\operatorname{deg} g=n$ and $\operatorname{deg} f=n-d, d \geq 1$, and let $p$ be a prime number that divides none of the leading coefficients of $f$ and $g$. Then the polynomial $f(X)+p^{k} g(X)$ is irreducible over $\mathbb{Q}$ for all but finitely many positive integers $k$ that are prime to $d$.

For the proof of our results we will need the following lemma, which might be of independent interest.

Lemma 1.4. Let $f, g \in \mathbb{Z}[X]$ be two polynomials with $\operatorname{deg} g=n$ and $\operatorname{deg} f=n-d, d \geq 1$. Let also $p$ be a prime number that divides none of the leading coefficients of $f$ and $g$, and let $k$ be any positive integer prime to $d$. If $f(X)+p^{k} g(X)$ may be written as a product of two non-constant polynomials with integer coefficients, say $f_{1}$ and $f_{2}$, then one of the leading coefficients of $f_{1}$ and $f_{2}$ must be divisible by $p^{k}$.

\section{Proof of the Main Results}

We will first recall some facts about Newton polygons (see for instance [1]), that will be required in the proof of Lemma 1.4. So let $p$ be a fixed prime number, and let $f(X)=$ $\sum_{i=0}^{n} a_{i} X^{i}$ be a polynomial with integer coefficients, $a_{0} a_{n} \neq 0$. Let us represent now the non-zero coefficients of $f$ in the form $a_{i}=\alpha_{i} p^{\beta_{i}}$, where $\alpha_{i}$ is an integer not divisible by $p$, and let us assign to each non-zero coefficient $\alpha_{i} p^{\beta_{i}}$ a point in the plane with integer coordinates $\left(i, \beta_{i}\right)$. The Newton polygon (or the Newton diagram) of $f$ corresponding to the prime $p$ is 
constructed from these points as follows. Let $P_{0}=\left(0, \beta_{0}\right)$ and let $P_{1}=\left(i_{1}, \beta_{i_{1}}\right)$, where $i_{1}$ is the largest integer for which there are no points $\left(i, \beta_{i}\right)$ below the segment $P_{0} P_{1}$. Next, let $P_{2}=\left(i_{2}, \beta_{i_{2}}\right)$, where $i_{2}$ is the largest integer for which there are no points $\left(i, \beta_{i}\right)$ below the segment $P_{1} P_{2}$, and so on (see Figure 1). The very last segment that we will draw will be $P_{m-1} P_{m}$, say, where $P_{m}=\left(n, \beta_{n}\right)$. We note that the broken line constructed so far is the lower convex hull of the points $\left(i, \beta_{i}\right), i=0, \ldots, n$. Now, if some segments of the broken line $P_{0} P_{1} \ldots P_{m}$ pass through points in the plane that have integer coordinates, then such points in the plane will be also considered as vertices of the broken line. In this way, to the vertices $P_{0}, P_{1}, \ldots, P_{m}$ plotted in the first phase, we might need to add a number of $s \geq 0$ more vertices.

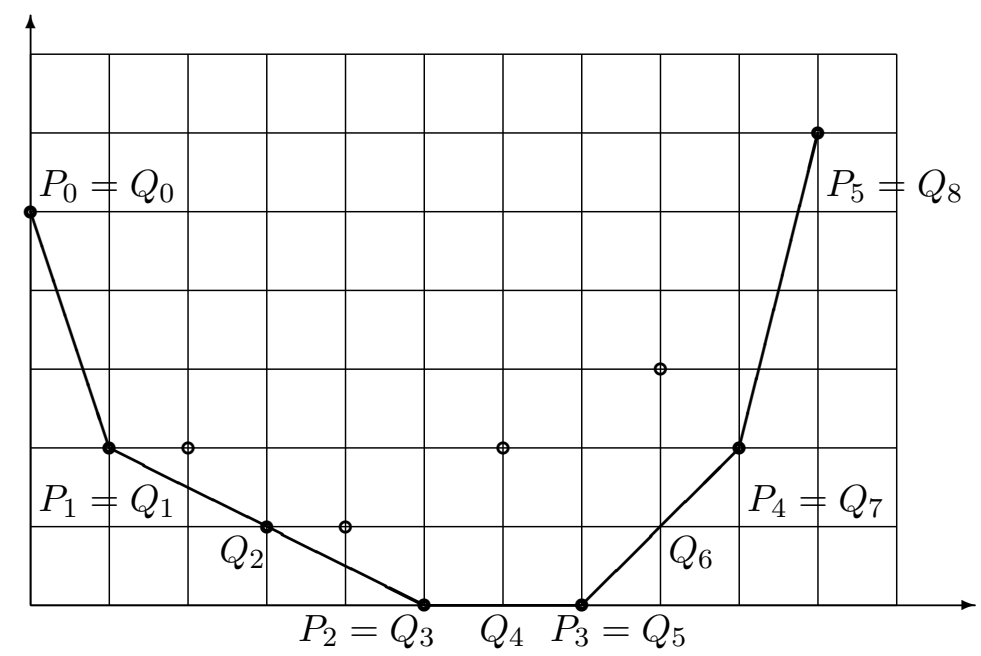

Figure 1. The Newton polygon of $f(X)=2^{5}+2^{2} X+2^{2} X^{2}+2 X^{3}+2 X^{4}+3 X^{5}+2^{2} X^{6}-5 X^{7}+$ $2^{3} X^{8}+2^{2} X^{9}+2^{6} X^{10}$ with respect to $p=2$.

The Newton polygon of $f$ (with respect to the prime number $p$ ) is the resulting broken line $Q_{0} Q_{1} \ldots Q_{m+s}$ obtained after relabelling all these points from left to the right, as they appear in this construction (here $Q_{0}=P_{0}$ and $Q_{m+s}=P_{m}$ ). With this notation, $P_{j} P_{j+1}$ and $Q_{i} Q_{i+1}$ are called edges and segments of the Newton polygon, respectively, and the vectors $\overrightarrow{Q_{i} Q_{i+1}}$ are called the vectors of the segments of the Newton polygon. Therefore, a segment $Q_{i} Q_{i+1}$ of the Newton polygon contains no points with integer coordinates other than $Q_{i}$ and $Q_{i+1}$. The collection of the vectors of the segments of the Newton polygon, taking each vector with its multiplicity, that is as many times as it appears, is called the system of vectors for the Newton polygon. With these definitions, we have the following celebrated result of Dumas [7], which plays a central role in the theory of Newton polygons.

Theorem (Dumas). Let $p$ be a prime number and let $f=g h$, where $f, g$ and $h$ are polynomials with integer coefficients. Then the system of vectors of the segments for the Newton polygon of $f$ with respect to $p$ is the union of the systems of vectors of the segments for the Newton polygons of $g$ and $h$ with respect to $p$. 
In other words, Dumas' Theorem says that the edges in the Newton polygon of $f=g h$ with respect to $p$ are formed by constructing polygonal path composed by translates of all the edges that appear in the Newton polygons of $g$ and $h$ with respect to $p$, using exactly one translate for each edge, in such a way as to form a polygonal path with the slopes of the edges increasing.

For applications of Newton's polygon method in the study of the irreducibility for various classes of polynomials, like for instance Bessel polynomials and Laguerre polynomials, we refer the reader to the work of Filaseta [9], [8], Filaseta, Kidd and Trifonov [11], Filaseta, Finch and Leidy [10], Filaseta and Lam [12], Filaseta and Trifonov [13], Filaseta and Williams [14], Hajir [15], [16], [17], and Sell [18].

Proof of Lemma 1.4. Let $f(X)=a_{0}+a_{1} X+\cdots+a_{n-d} X^{n-d}$ and $g(X)=b_{0}+b_{1} X+$ $\cdots+b_{n} X^{n}, a_{n-d} b_{n} \neq 0$, and let us write $f(X)+p^{k} g(X)=c_{0}+c_{1} X+\cdots+c_{n} X^{n}$, where $c_{i}=a_{i}+p^{k} b_{i}$ for $i=0, \ldots, n-d$, while $c_{i}=p^{k} b_{i}$ for $i=n-d+1, \ldots, n$. Now, since by our assumption $p \nmid a_{n-d} b_{n}$, we see that $c_{n-d}$ is not divisible by $p$, while $c_{n-d+1}, \ldots, c_{n}$ are all divisible by $p^{k}$, and moreover, $p^{k+1} \nmid c_{n}$. Therefore, in the Newton polygon of $f+p^{k} g$ with respect to the prime number $p$, the right-most edge will join the points $(n-d, 0)$ and $(n, k)$, labelled in Figure 2 below by $Q_{m}$ and $Q_{m-1}$, respectively.

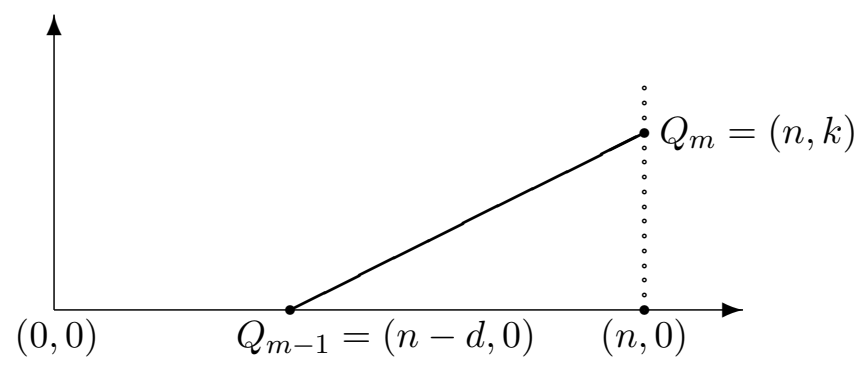

Figure 2. The right-most segment in the Newton polygon of $f(X)+p^{k} g(X)$ with respect to $p$.

Moreover, since $k$ is prime to $d$, one may easily deduce that $Q_{m-1} Q_{m}$ is in fact a segment of the Newton polygon, since it contains no points with integer coordinates other than the points $Q_{m-1}$ and $Q_{m}$. Indeed, the equation of the straight line passing through $Q_{m-1}$ and $Q_{m}$ is given by

$$
y=\frac{k}{d} x+\frac{k}{d}(d-n)
$$

and, for $j=1, \ldots, d-1$, the $y$-coordinate of the point $(n-j, k(d-j) / d)$ on this line is never a rational integer since $k$ and $d$ are coprime. Now, since the sequence of the slopes of the edges of the Newton polygon, when considered from left to the right, is strictly increasing, we deduce that $Q_{m-1} Q_{m}$ is the only segment with positive slope. On the other hand, if $f+p^{k} g$ may be written as a product of two non-constant polynomials $f_{1}$ and $f_{2}$ with integer coefficients, then each of the factors $f_{1}$ and $f_{2}$ must have at least one coefficient which is not divisible by $p$. If we assume now that each of the leading coefficients of $f_{1}$ and $f_{2}$ is divisible 
by $p$, then each of the Newton polygons of $f_{1}$ and $f_{2}$ with respect to $p$ would contain at least one segment with positive slope, thus producing by Dumas' Theorem at least two segments with positive slopes in the Newton polygon of $f+p^{k} g$, a contradiction. Therefore, one of the leading coefficients of $f_{1}$ and $f_{2}$ must be divisible by $p^{k}$, while the other must be coprime with $p$. This completes the proof of our lemma.

Proof of Theorem 1.1. We will adapt the ideas in [2] and [6]. The proof will be obtained by contradiction and will consist of two parts. In the first part we will prove that if our polynomial factorizes as $f(X)+p^{k} g(X)=f_{1}(X) f_{2}(X)$, then one of the leading coefficients of the hypothetical factors $f_{1}$ and $f_{2}$, say the leading coefficient of $f_{1}$, will not exceed in modulus the modulus of the leading coefficient of $g$. The second part will consist in proving that the conclusion of the first part forces the resultant $R\left(g, f_{1}\right)$ to be less than 1 in absolute value, which obviously can not hold.

Let $f(X)=a_{0}+a_{1} X+\cdots+a_{n-d} X^{n-d}$ and $g(X)=b_{0}+b_{1} X+\cdots+b_{n} X^{n}$ be two relatively prime polynomials with integer coefficients, $a_{n-d} b_{n} \neq 0$, and let $p$ be a prime number that divides not $a_{n-d} b_{n}$, and $k$ a positive integer prime to $d$, such that

$$
p^{k} \geq\left(2+\frac{1}{2^{n+1-d} H(g)^{n+1}}\right)^{n+1-d} H(f) H(g)^{n}-\frac{H(f)}{H(g)} .
$$

We may obviously assume that $n \geq d+1$. Now let us assume that the polynomial $f(X)+$ $p^{k} g(X)$ is reducible, that is

$$
f(X)+p^{k} g(X)=f_{1}(X) f_{2}(X),
$$

with $f_{1}(X), f_{2}(X) \in \mathbb{Z}[X]$ and $\operatorname{deg} f_{1} \geq 1, \operatorname{deg} f_{2} \geq 1$, say

$$
\begin{aligned}
& f_{1}(X)=c_{0}+c_{1} X+\cdots+c_{s} X^{s}, \\
& f_{2}(X)=d_{0}+d_{1} X+\cdots+d_{t} X^{t},
\end{aligned}
$$

$c_{0}, \ldots c_{s}, d_{0}, \ldots d_{t} \in \mathbb{Z}, c_{s} d_{t} \neq 0$, and $s \geq 1, t \geq 1, s+t=n$. By Lemma 1.4 we see that one of the leading coefficients of $f_{1}$ and $f_{2}$ will be divisible by $p^{k}$, and the other one will be prime to $p$. Without loss of generality we may assume that $p^{k} \mid d_{t}$ and $p \nmid c_{s}$, hence $c_{s}$ must be a divisor of $b_{n}$. In particular, we must have

$$
\left|c_{s}\right| \leq\left|b_{n}\right| .
$$

Note that for $k=1$, the condition $p \nmid a_{n-d}$ is an immediate consequence of (1), which also yields $p \nmid b_{n}$. To prove this claim, we only need to observe that

$$
\left(2+\frac{1}{2^{n+1-d} H(g)^{n+1}}\right)^{n+1-d} H(f) H(g)^{n}-\frac{H(f)}{H(g)}>H(f)(2 H(g)-1) \geq H(f) H(g) .
$$

Also note that for $k=1$ the inequality (2) follows from the equality $c_{s} d_{t}=p b_{n}$ and the fact that $p \nmid b_{n}$. 
Now we are going to estimate the resultant $R\left(g, f_{1}\right)$. Since $g$ and $f_{1}$ are relatively prime polynomials, $R\left(g, f_{1}\right)$ must be a nonzero rational integer, so in particular we have

$$
\left|R\left(g, f_{1}\right)\right| \geq 1
$$

If we decompose $f_{1}$, say $f_{1}(X)=c_{s}\left(X-\theta_{1}\right) \cdots\left(X-\theta_{s}\right)$, with $\theta_{1}, \ldots, \theta_{s} \in \mathbb{C}$, then

$$
\left|R\left(g, f_{1}\right)\right|=\left|c_{s}\right|^{n} \prod_{1 \leq j \leq s}\left|g\left(\theta_{j}\right)\right| .
$$

Since each root $\theta_{j}$ of $f_{1}$ is also a root of $f(X)+p^{k} g(X)$, we have

$$
g\left(\theta_{j}\right)=-\frac{f\left(\theta_{j}\right)}{p^{k}}
$$

and moreover, since $f$ and $g$ are relatively prime, $f\left(\theta_{j}\right) \neq 0$ and $g\left(\theta_{j}\right) \neq 0$ for any index $j \in\{1, \ldots, s\}$. Using now (4) and (5), we obtain

$$
\left|R\left(g, f_{1}\right)\right|=\frac{\left|c_{s}\right|^{n}}{p^{k s}} \prod_{1 \leq j \leq s}\left|f\left(\theta_{j}\right)\right|
$$

We now proceed to find an upper bound for $\left|f\left(\theta_{j}\right)\right|$. The equality $f\left(\theta_{j}\right)+p^{k} g\left(\theta_{j}\right)=0$ implies

$$
\left(a_{0}+p^{k} b_{0}\right)+\left(a_{1}+p^{k} b_{1}\right) \theta_{j}+\cdots+\left(a_{n-d}+p^{k} b_{n-d}\right) \theta_{j}^{n-d}+p^{k} b_{n-d+1} \theta_{j}^{n-d+1}+\cdots+p^{k} b_{n} \theta_{j}^{n}=0,
$$

from which we deduce that

$$
\begin{aligned}
p^{k}\left|b_{n}\right| \cdot\left|\theta_{j}\right|^{n \leq} \leq\left|a_{0}\right|+p^{k}\left|b_{0}\right| & +\left(\left|a_{1}\right|+p^{k}\left|b_{1}\right|\right) \cdot\left|\theta_{j}\right|+\cdots+\left(\left|a_{n-d}\right|+p^{k}\left|b_{n-d}\right|\right) \cdot\left|\theta_{j}\right|^{n-d} \\
& \quad+p^{k}\left|b_{n-d+1}\right| \cdot\left|\theta_{j}\right|^{n-d+1}+\cdots+\cdots+p^{k}\left|b_{n}\right| \cdot\left|\theta_{j}\right|^{n} \\
\leq & \left(H(f)+p^{k} H(g)\right)\left(1+\left|\theta_{j}\right|+\cdots+\left|\theta_{j}\right|^{n-1}\right) .
\end{aligned}
$$

For $\left|\theta_{j}\right|>1$ we find

$$
p^{k}\left|b_{n}\right| \cdot\left|\theta_{j}\right|^{n}<\left(H(f)+p^{k} H(g)\right) \cdot \frac{\left|\theta_{j}\right|^{n}}{\left|\theta_{j}\right|-1},
$$

whence

$$
\left|\theta_{j}\right|<1+\frac{1}{\left|b_{n}\right|} \cdot\left(\frac{H(f)}{p^{k}}+H(g)\right) .
$$

Notice that inequality (7) is trivially true when $\left|\theta_{j}\right| \leq 1$. Since obviously

$$
\left|f\left(\theta_{j}\right)\right| \leq H(f) \cdot\left(1+\left|\theta_{j}\right|+\cdots+\left|\theta_{j}\right|^{n-d}\right)
$$

inequality (7) yields

$$
\left|f\left(\theta_{j}\right)\right|<H(f) \cdot \frac{\left[1+\frac{1}{\left|b_{n}\right|} \cdot\left(\frac{H(f)}{p^{k}}+H(g)\right)\right]^{n+1-d}-1}{\frac{1}{\left|b_{n}\right|} \cdot\left(\frac{H(f)}{p^{k}}+H(g)\right)} .
$$


Using now (6) and (8), we obtain

$$
\left|R\left(g, f_{1}\right)\right|<\frac{\left|c_{s}\right|^{n}}{p^{k s}}\left[\left|b_{n}\right| H(f) \cdot \frac{\left[1+\frac{1}{\left|b_{n}\right|} \cdot\left(\frac{H(f)}{p^{k}}+H(g)\right)\right]^{n+1-d}-1}{\frac{H(f)}{p^{k}}+H(g)}\right]^{s} .
$$

Since $s \geq 1$ implies $\left|c_{s}\right|^{n / s} \leq\left|c_{s}\right|^{n}$, all we need to prove is that our assumption on $p^{k}$ will force

$$
\frac{\left|c_{s}\right|^{n}}{p^{k}} \cdot\left|b_{n}\right| H(f) \cdot \frac{\left[1+\frac{1}{\left|b_{n}\right|} \cdot\left(\frac{H(f)}{p^{k}}+H(g)\right)\right]^{n+1-d}-1}{\frac{H(f)}{p^{k}}+H(g)} \leq 1
$$

Using (2), it will be sufficient to prove that

$$
\left|b_{n}\right|^{n+1}\left[1+\frac{1}{\left|b_{n}\right|} \cdot\left(\frac{H(f)}{p^{k}}+H(g)\right)\right]^{n+1-d} \leq 1+\left|b_{n}\right|^{n+1}+\frac{p^{k} H(g)}{H(f)},
$$

which is equivalent to

$$
\left|b_{n}\right|^{d}\left[\left|b_{n}\right|+\frac{H(f)}{p^{k}}+H(g)\right]^{n+1-d} \leq 1+\left|b_{n}\right|^{n+1}+\frac{p^{k} H(g)}{H(f)} .
$$

Now, since $\left|b_{n}\right| \leq H(g)$, it suffices to prove that

$$
H(g)^{d}\left[2 H(g)+\frac{H(f)}{p^{k}}\right]^{n+1-d} \leq 1+\frac{p^{k} H(g)}{H(f)},
$$

or equivalently, that

$$
p^{k} \geq H(f) H(g)^{n}\left[2+\frac{H(f)}{p^{k} H(g)}\right]^{n+1-d}-\frac{H(f)}{H(g)} .
$$

If we consider the function

$$
\mathcal{F}(x):=H(f) H(g)^{n}\left[2+\frac{H(f)}{x H(g)}\right]^{n+1-d}-\frac{H(f)}{H(g)} \quad \text { for } x>0,
$$

then in view of (9) we have to search for a value of $p^{k}$ as small as possible such that $p^{k} \geq \mathcal{F}\left(p^{k}\right)$. In this respect, since $\mathcal{F}$ is a decreasing function, it will be sufficient to search for a suitable $\delta>0$, such that

$$
p^{k} \geq \delta H(f) H(g)^{n}-\frac{H(f)}{H(g)}
$$

and

$$
\delta H(f) H(g)^{n}-\frac{H(f)}{H(g)} \geq \mathcal{F}\left(\delta H(f) H(g)^{n}-\frac{H(f)}{H(g)}\right) .
$$

Therefore it will be sufficient to find a positive $\delta$ as small as possible satisfying

$$
\delta H(f) H(g)^{n}-\frac{H(f)}{H(g)} \geq H(f) H(g)^{n}\left(2+\frac{H(f)}{\left(\delta H(f) H(g)^{n}-\frac{H(f)}{H(g)}\right) H(g)}\right)^{n+1-d}-\frac{H(f)}{H(g)},
$$


that is

$$
\delta \geq\left(2+\frac{1}{\delta \cdot H(g)^{n+1}-1}\right)^{n+1-d} .
$$

A suitable candidate for $\delta$ is easily seen to be $\left(2+2^{d-n-1} H(g)^{-n-1}\right)^{n+1-d}$, since

$$
\left(2+\frac{1}{2^{n+1-d} H(g)^{n+1}}\right)^{n+1-d}>\left(2+\frac{1}{\left(2+2^{d-n-1} H(g)^{-n-1}\right)^{n+1-d} H(g)^{n+1}-1}\right)^{n+1-d}
$$

for $n \geq d+1$. Indeed, (10) is equivalent to

$$
\left(2+\frac{1}{2^{n+1-d} H(g)^{n+1}}\right)^{n+1-d} H(g)^{n+1}-2^{n+1-d} H(g)^{n+1}>1
$$

which obviously holds for $n \geq d+1$, since

$$
\begin{aligned}
\left(2+\frac{1}{2^{n+1-d} H(g)^{n+1}}\right)^{n+1-d}-2^{n+1-d} & =\frac{1}{2^{n+1-d} H(g)^{n+1}} \sum_{i=0}^{n-d} 2^{i}\left(2+\frac{1}{2^{n+1-d} H(g)^{n+1}}\right)^{n-d-i} \\
& >\frac{1}{2^{n+1-d} H(g)^{n+1}} \sum_{i=0}^{n-d} 2^{i} 2^{n-d-i}=\frac{n+1-d}{2 H(g)^{n+1}}
\end{aligned}
$$

This proves that for

$$
p^{k} \geq\left(2+\frac{1}{2^{n+1-d} H(g)^{n+1}}\right)^{n+1-d} H(f) H(g)^{n}-\frac{H(f)}{H(g)}
$$

we have $\left|R\left(g, f_{1}\right)\right|<1$, which contradicts (3). This completes the proof of the theorem.

Remark 2.1. We note here that the conclusion of Theorem 1.1 also holds if $\operatorname{deg} f=0$, that is, when $f$ is a nonzero constant polynomial. Moreover, in this case we do not need to ask that $p^{k}$ exceeds a certain lower bound, as in the statement of Theorem 1.1. Here $d=n$ and $f=a_{0}$, say, with $p \nmid a_{0} b_{n}$, and we only need to ask $k$ and $n$ to be relatively prime. The reason is that in this particular case the Newton polygon of $f+p^{k} g$ consists of a single segment that joins the points $(0,0)$ and $(n, k)$, and the irreducibility of $f+p^{k} g$ is a direct consequence of Dumas' Theorem.

Remark 2.2. We end by noting that one may easily obtain sharper results, if some additional information on the coefficients or on the roots of $f$ and $g$ is available. For instance, using the notation from the proof of Theorem 1.1, if

$$
\left|b_{n}\right| \geq \sum_{i=0}^{n-1}\left|b_{i}\right|+\sum_{i=0}^{n-d}\left|a_{i}\right|
$$

then we also have

$$
p^{k}\left|b_{n}\right| \geq \sum_{i=0}^{n-d}\left|a_{i}+p^{k} b_{i}\right|+\sum_{i=n-d+1}^{n-1} p^{k}\left|b_{i}\right|
$$


so all the roots $\theta$ of $f(X)+p^{k} g(X)$ satisfy $|\theta| \leq 1$. Then we have

$$
\left|R\left(g, f_{1}\right)\right|=\frac{\left|c_{s}\right|^{n}}{p^{k s}} \prod_{1 \leq j \leq s}\left|f\left(\theta_{j}\right)\right| \leq \frac{\left|b_{n}\right|^{n}\left(\left|a_{0}\right|+\cdots+\left|a_{n-d}\right|\right)^{s}}{p^{k s}} .
$$

Therefore, if $p^{k}>\left|b_{n}\right|^{n}\left(\left|a_{0}\right|+\cdots+\left|a_{n-d}\right|\right)$, then $f(X)+p^{k} g(X)$ is irreducible over $\mathbb{Q}$.

It is also worth mentioning that in case $\left|b_{n}\right|<H(g)$, the conclusion in Theorem 1.1 holds for $p^{k} \geq 2^{n+1-d} H(f) H(g)^{n}$. Indeed, since $p^{k}>H(f)$ and $\left|b_{n}\right|+1 \leq H(g)$, we have $\left|b_{n}\right|+\frac{H(f)}{p^{k}}+H(g)<2 H(g)$, so instead of inequality (9) we arrive at

$$
p^{k} \geq 2^{n+1-d} H(f) H(g)^{n}-\frac{H(f)}{H(g)} .
$$

Acknowledgements. This work was partially supported by a LEA Math-Mode project. We are grateful to an anonymous referee, who helped us to improve the results of this paper.

\section{REFERENCES}

[1] E.J. Barbeau, Polynomials, Problem Books in Mathematics, Springer (2003), 455 p.

[2] N.C. Bonciocat, Upper bounds for the number of factors for a class of polynomials with rational coeffcients, Acta Arith. 113 (2004), 175-187.

[3] A.I. Bonciocat, N.C. Bonciocat, A. Zaharescu, On the number of factors of convolutions of polynomials with integer coefficients, Rocky Mountain J. Math. 38 (2008), 417-431.

[4] A.I. Bonciocat, A. Zaharescu, Irreducibility results for compositions of polynomials with integer coefficients, Monatsh. Math. 149 (2006), 31-41.

[5] M. Cavachi, On a special case of Hilbert's irreducibility theorem, J. Number Theory 82 (2000), 96-99.

[6] M. Cavachi, M. Vâjâitu, A. Zaharescu, A class of irreducible polynomials, J. Ramanujan Math. Soc. 17 (2002), 161-172.

[7] G. Dumas, Sur quelques cas d'irreductibilité des polynômes à coefficients rationnels, Journal de Math. Pures et Appl. 2 (1906), 191-258.

[8] M. Filaseta, The irreducibility of all but finitely many Bessel polynomials, Acta Math. 174 (1995), 383397.

[9] M. Filaseta, A generalization of an irreducibility theorem of I. Schur, in: Analytic Number Theory, Proc. Conf. in Honor of Heini Halberstam, vol. 1, (B.C. Berndt, H.G. Diamond, and A.J. Hildebrand, eds.), Birkhäuser, Boston, 1996, pp. 371-395.

[10] M. Filaseta, C. Finch, J.R. Leidy, T.N. Shorey's influence in the theory of irreducible polynomials, in: Diophantine Equations (ed. N. Saradha), Narosa Publ. House, New Delhi, 2005, pp. 77-102.

[11] M. Filaseta, T. Kidd, O. Trifonov, Laguerre polynomials with Galois group $A_{m}$ for each $m$, J. Number Theory 132 (2012), 776-805.

[12] M. Filaseta, T.-Y. Lam, On the irreducibility of the generalized Laguerre polynomials, Acta Arth. 105 (2002), 177-182.

[13] M. Filaseta, O. Trifonov, The Irreducibility of the Bessel polynomials, J. Reine Angew. Math. 550 (2002), $125-140$.

[14] M. Filaseta, R.L. Williams, Jr., On the irreducibility of a certain class of Laguerre polynomials, J. Number Theory 100 (2003), 229-250.

[15] F. Hajir, Some $A_{n}$-extensions obtained from generalized Laguerre polynomials, J. Number Theory 50 (1995), 206-212. 
[16] F. Hajir, On the Galois group of generalized Laguerre polynomials, J. Théor. Nombres Bordeaux 17 (2005), 517-525.

[17] F. Hajir, Algebraic properties of a family of generalized Laguerre polynomials, Canad J. Math. 61 (2009) no. $3,583-603$.

[18] E.A. Sell, On a certain family of generalized Laguerre polynomials, J. Number Theory 107 (2004), 266281.

Simion Stollow Institute of Mathematics of the Romanian Academy, Research Unit 5, P.O. Box 1-764, Bucharest 014700, Romania

E-mail address: Nicolae.Bonciocat@imar.ro, Mihai.Cipu@imar.ro

Université de Strasbourg, Mathématiques, 7, rue René Descartes, 67084 Strasbourg Cedex, FRANCE

E-mail address: yann.bugeaud@math.unistra.fr, maurice.mignotte@math.unistra.fr 